\title{
Amino acids and poly(amino acids) as nucleating agents for poly(lactic acid)
}

\begin{abstract}
In industry, rapid crystallization is often required. At present, the crystallization rate of poly(lactic acid) (PLA) is too low compared to industrial needs. In this paper, amino acids such as glycine and L-alanine and poly(amino acids) like polyglycine and poly-DL-alanine are considered as heterogeneous crystallization nucleating agents. The impact of adding these bio-based additives on the isothermal crystallization behavior of PLA was quantified and compared at different concentrations by using the so-called Lotz efficiency scale, which here is based on isothermal DSC measurements. In addition, rheological and rheo-optical techniques were used to monitor the isothermal crystallization. Our results indicate that polyglycine possesses a significant nucleating ability $(60.5 \%)$ which is comparable to the industrially used talc (81.1\%).
\end{abstract}

Keywords: amino acids; isothermal crystallization; nucleating agent; PLA; poly(amino acids).

DOI 10.1515/polyeng-2014-0175

Received June 26, 2014; accepted August 6, 2014; previously published online September 4, 2014

\section{Introduction}

Synthetic polymers have been successfully developed from petroleum-based products for decades and their crystallization behavior has been intensively studied $[1,2]$. However, the use of these polymers leads to severe problems including overexploitation of fossil resources and environmental pollution. Therefore, there is a growing

\footnotetext{
*Corresponding author: Maria Josefina Carbone, Soft Matter, Applied Rheology and Technology, Department of Chemical Engineering, KU Leuven, Willem de Croylaan 46, Leuven, 3001, Belgium, e-mail: josefina.carbone@cit.kuleuven.be

Maja Vanhalle and Bart Goderis: Polymer Chemistry and Materials, Department of Chemistry, KU Leuven, Celestijnenlaan 200F, Leuven, 3001, Belgium

Peter Van Puyvelde: Soft Matter, Applied Rheology and Technology, Department of Chemical Engineering, KU Leuven, Willem de Croylaan 46, Leuven, 3001, Belgium
}

interest in the development of bio-based alternatives, that for some purposes also deserve being biodegradable [3-5]. Poly(lactic acid) (PLA) is a synthetic thermoplastic aliphatic polyester with interesting properties. It can be produced from renewable resources, it is biodegradable and compostable [6]. PLA still needs to be improved in order to compete with petroleum-based polymers and, hereto, extend potential applications. Aspects such as low melt strength, restricted thermal and barrier properties and extremely low crystallization rates reduce PLA processing possibilities and application window. This paper focuses on enhancing PLA crystallization rate, since high rates are often needed in industry [5].

The crystallization behavior of PLA has been addressed by many research groups. In 1996, Iannace and Nicolais [7] used DSC and dielectric measurements to examine the isothermal crystallization kinetics from the melt at crystallization temperatures $\left(\mathrm{T}_{\mathrm{c}}\right.$ ) between $90^{\circ} \mathrm{C}$ and $135^{\circ} \mathrm{C}$. Some years later, Di Lorenzo [8] observed a sudden acceleration of the PLA crystallization kinetics between $110^{\circ} \mathrm{C}$ and $120^{\circ} \mathrm{C}$. This phenomenon gives rise to a discontinuity in the typical bell-shaped curve where the characteristic crystallization time is plotted as a function of $\mathrm{T}_{\mathrm{c}}$. Yasuniwa et al. [9] explained it as a change in crystal structure from orthorhombic ( $\alpha$-form) to trigonal ( $\beta$-form), formed at high and low $\mathrm{T}_{c} \mathrm{~s}$, respectively. Furthermore, Miyata and Masuko [10] and He et al. [11] investigated the influence of PLA molecular weight on the isothermal crystallization behavior. They agreed that the crystallization rate decreases with increasing molecular weight $\left(50 \mathrm{~kg} \mathrm{~mol}^{-1}\right.$, $100 \mathrm{~kg} \mathrm{~mol}^{-1}, 200 \mathrm{~kg} \mathrm{~mol}^{-1}$ ), as was expected from results of other crystallizable polymers.

The lactic acid molecule contains a chiral C atom, resulting in two optical isomers, L- lactic acid and D-lactic acid, and three lactide versions, D,D-lactide, L,L-lactide and D,L-lactide or meso-lactide. Consequently, after polymerization, poly(L-lactic acid) (PLLA), poly(D-lactic acid) (PDLA) and poly(DL-lactic acid) can be obtained depending on the starting stereochemical composition of lactide monomers. This determines the final properties of the polymer [5]. Huang et al. [12] showed that when the D-lactic acid isomer composition in an $\mathrm{L}$ chain increases, the $T_{c}$ at maximal crystal growth rate decreases, indicating that samples with a higher D-isomer concentration 
crystallize more slowly. In 2005, Tsuji et al. [13] completed this study and described the transition of growth rate regimes.

In industry, heterogeneous nucleating agents are commonly used to enhance the rate of crystallization and to increase the degree of crystallinity in many polymers including PLA. Different research groups have experimented with diverse nucleating agents, but many of them concentrated on using stereocomplexes. PLA exhibits the interesting property of forming stereocomplexes from enantiomeric blends of PLLA and PDLA with a melting temperature much higher than the constituting components [14-20].

Schmidt and Hillmyer [15] claimed that PLA stereocomplex crystallites are formed in situ when PDLA is added to PLLA and act as effective heterogeneous nucleating agents due to favorable interactions between the polymer melt and these stereocomplex crystal fragments. They calculated the non-isothermal crystallization efficiency for PLLA (83.3 and $94.0 \mathrm{~kg} \mathrm{~mol}^{-1}$ ) when $1 \mathrm{wt} \%$ PDLA (14.6 kg mol $^{-1}$ ) or $1 \mathrm{wt} \%$ talc was added: the former scored $36 \%$ and the latter $28 \%$. These efficiencies were calculated by using a DSC based methodology which is called the Lotz scale further on. In the case of isothermal experiments at $140^{\circ} \mathrm{C}$, there was a much larger difference: the PDLA nucleated sample reached its half crystallization after $249 \mathrm{~s}$, while it took $2232 \mathrm{~s}$ for the talc nucleated sample [15].

Yamane and Sasai [16] investigated the effect of PDLA content (1-5 wt\%) and molecular weight on the crystallization behavior and thermal properties of PLLA. In a blend of PLLA with a low amount of low molecular weight PDLA, which was heated above the stereocomplex melting temperature and then cooled down, there was no crystallization enhancement due to the PDLA molecules short length and restricted surface area to nucleate. Only when PDLA concentration was increased, could stereocomplex crystallites be formed and act as nucleating sites. Later, non-isothermal Lotz nucleating efficiencies (cooled at $5^{\circ} \mathrm{C} \mathrm{min}^{-1}$ ) close to $100 \%$ were obtained by optimizing molecular weight and concentration of the added PDLA (14 $\mathrm{kg} \mathrm{mol}^{-1}$, $3 \mathrm{wt} \%$ ). In the case of samples which were isothermally crystallized at $140^{\circ} \mathrm{C}$, the half crystallization times were less than $60 \mathrm{~s}[14,19]$.

After comparing different industrially available nucleating agents, it appeared that talc is the most efficient, beside stereocomplex crystallites [14, 17, 21, 22]. Tsuji et al. [17] proposed the following gradation of nucleation efficiency in PLLA $\left(\mathrm{T}_{\mathrm{c}}=115.9^{\circ} \mathrm{C}\right)$ when $3 \mathrm{wt} \%$ of nucleating agent was added: $52 \mathrm{~kg} \mathrm{~mol}^{-1}$ PDLA $\left(\mathrm{T}_{\mathrm{c}}=143.9^{\circ} \mathrm{C}\right)>$ talc $\left(\mathrm{T}_{\mathrm{c}}=128.3^{\circ} \mathrm{C}\right)>$ fullerenes $\left(\mathrm{T}_{\mathrm{c}}=126.9^{\circ} \mathrm{C}\right)>$ montmorillonite $\left(\mathrm{T}_{\mathrm{c}}=120.4^{\circ} \mathrm{C}\right)$. Furthermore, other research groups focused on developing new additives such as self-assembling or chemical nucleating agents [23] like $\mathrm{CaCO} 3, \mathrm{TiO} 2, \mathrm{BaSO} 4$ [24], hydrazide compounds [25] or ethylene-bis-stearamide [26].

Since the nucleation efficiency is highly related to chemical and crystallographic matching between nucleating agent and matrix polymer, it makes sense to look for molecules with a chemical structure similar to PLA. In this logic, the group of Tsuji et al. [27, 28] proposed to use other types of biodegradable polyesters as crystallization enhancing agents for PLA. Concentrations between $0.1 \mathrm{wt} \%$ and $10 \mathrm{wt} \%$ of poly[(R)-3-hydroxybutyrate] (PHB), poly( $\varepsilon$-caprolactone) (PCL) or poly (glycolic acid) (PGA) were added to a PLA matrix and it was confirmed that under certain conditions and concentrations, they have a significant effect on PLA crystallization. PCL and PGA were termed nucleation assisting agents and PHB, a spherulite growth-accelerating agent.

One of the many advantages of PLA, with respect to other polymers, is its biodegradability, which in addition to its biocompatibility makes it suitable for a large range of applications. Therefore, additives have to be chosen carefully to ensure that these bio-advantages are preserved while the final material properties can still be tailored.

Pan et al. [29] examined the improvement of the crystallization behavior by solvent mixing PLLA with nucleobases (concentrations ranging from $0.1 \mathrm{wt} \%$ to $10 \mathrm{wt} \%$ ), such as cytosine, guanine, adenine, thymine and uracil, which all are building blocks of deoxyribonucleic acid (DNA). They studied their effects on the isothermal and non-isothermal crystallization of PLA and the morphology of the resulting crystals. Most nucleobases had a limited effect, while with $1 \mathrm{wt} \%$ of uracil, enhanced crystallization rates were clearly observed. Furthermore, Tachibana and co-workers [30] tested myo-inositol as a nucleating agent and proved that it promoted PLA isothermal crystallization already at low concentrations (1-5 wt \%). Accelerated PLA isothermal crystallization was also observed in the case of $1 \mathrm{wt} \%$ partially silicated cellulose nanocrystals/PLLA microcomposite films. However, the addition of these nano-particles had an unfavorable effect on the mechanical properties because of poor interfacial PLLA-nanocrystals adhesion [31].

Until now, the use of amino acids or poly(amino acids), also called polypeptides, as nucleating agents has not been studied. The addition of poly(amino acids) as heterogeneous nucleating agents makes sense, given that their chemical structure is similar to that of PLA, which may facilitate the crystal nuclei formation. Furthermore, they do not compromise PLA's bio-advantages. In addition to the use of poly(amino acids), the addition of single amino acids was studied in this work as well. 
Table 1 Amino acids and poly(amino acids).

\begin{tabular}{llll}
\hline $\begin{array}{l}\text { Amino } \\
\text { acid }\end{array}$ & $\begin{array}{l}\text { Chemical } \\
\text { structure }\end{array}$ & $\begin{array}{l}\text { Poly(amino } \\
\text { acid) }\end{array}$ & $\begin{array}{l}\text { Chemical } \\
\text { structure }\end{array}$ \\
\hline L-Alanine & Polylanine & Polyglycine &
\end{tabular}

Amino acids are biological monomers, which have the same backbone structure with a basic $\mathrm{NH}_{2}$ group and an acidic $\mathrm{COOH}$ group, but differ in side chains. Table 1 shows the structures of alanine and glycine. All amino acids, except glycine, contain a chiral $\mathrm{C}$ atom and thus are optically active, just like PLA. In nature, mainly the L-isomer is found [32].

The particular choice of alanine, polyalanine, glycine and polyglycine was based on preliminary results obtained from thermodynamic simulations, based on the Flory-Huggins model, performed to predict the miscibility level of PLA with different poly(amino acids). This study predicted that combinations of PLA with polyglycine or polyalanine were the most favorable, presumably because of their simple structure and limited steric hindrance [33].

The main objective of this paper is to perform a systematic study on the level of crystallization enhancement produced by the addition of amino acids and poly(amino acids), more specifically, L-alanine, poly-DL-alanine, glycine and polyglycine. Talc particles were used as reference nucleating agents, since literature pointed out that talc is one of the most efficient nucleating agents for PLA.

\section{Materials and methods}

\subsection{Materials and sample preparation}

PLA containing 4\% D enantiomer, supplied by Futerro (Belgium), was used as matrix material for this study. This PLA was mixed with the chosen nucleating agents by means of twin-screw extrusion. This method can induce some mechanical and thermal degradation, resulting in molecular weight reduction and accordingly, acceleration of crystallization, even when nitrogen is purged in the system. To distinguish between the effect of the extrusion step and the improvement caused by nucleating agents, neat PLA was also extruded and evaluated under the same
Table 2 Summary of poly(lactic acid) properties.

\begin{tabular}{lrrr}
\hline & $\mathbf{M}_{\mathrm{w}}\left(\mathbf{g ~ m o l}^{-1}\right)$ & $\mathbf{M}_{\mathrm{w}} / \mathbf{M}_{\mathrm{n}}(-)$ & $\boldsymbol{\eta}_{0}(\mathrm{~Pa} . \mathrm{s})$ \\
\hline Neat PLA & 119,567 & 1.7 & 70 \\
Extruded PLA & 116,091 & 1.7 & 67 \\
\hline
\end{tabular}

conditions as the other samples. The properties of the neat and extruded materials are compared in Table 2.

For this study, talc particles were used as the reference, since they are a common industrial nucleating agent and, as mentioned in literature, one of the most efficient nucleating agents for PLA [14, 17, 21, 22]. The particles used were $0.6 \mu \mathrm{m}(95 \%<3 \mu \mathrm{m})$, had a platelet shape and were not coated (Luzenac A3, Imerys Talc).

L-alanine was purchased at Applichem Merck with a molecular weight equal to $89 \mathrm{~g} \mathrm{~mol}^{-1}$ while glycine and polyglycine were purchased at Sigma Aldrich with a molecular weight of $75 \mathrm{~g} \mathrm{~mol}^{-1}$ and 500-5000 $\mathrm{g} \mathrm{mol}^{-1}$, respectively. No chiral purity determination was performed to the bought poly-DL-alanine (Sigma Aldrich) but it was known that the polymerization started from a racemic mixture (50:50 D, L isomers) resulting in a molecular weight between $1000 \mathrm{~g} \mathrm{~mol}^{-1}$ and $5000 \mathrm{~g} \mathrm{~mol}^{-1}$. All materials were used as received after drying and no purification steps were performed.

Prior to melt-mixing, PLA pellets and nucleating agents (powder) were dry-mixed in a container and deionized by using an antistatic kit (Mettler Toledo). Subsequently, they were melt-mixed at $200^{\circ} \mathrm{C}$ for $4 \mathrm{~min}$ using a $15 \mathrm{~cm}^{3}$ DSM micro-compounder (DSM Xplore) with a rotational speed of $100 \mathrm{rpm}$. The residence time could be chosen because the extruder had a recirculation channel. Mixing was performed under nitrogen atmosphere in order to reduce degradation of the polymer. The concentration of nucleating agent is given in wt $\%$, meaning the mass of the nucleating agent in mg divided by the total sample mass in mg (PLA+nucleating agent). Before each preparation step or experiment, all materials were dried overnight at $50^{\circ} \mathrm{C}$ under vacuum.

\subsection{Methods}

The microscopy study was performed with a Linkam shearing cell (Linkam Scientific) and a polarized light microscope (Laborlux 12 Pol S, Leitz or BX41 microscope, Olympus), equipped with a camera (C4742-95, Hamamatsu Photonics). The microscope images were directly digitalized by HiPic 9.2 (Hamamatsu Photonics) and analyzed with ImageJ. The measuring gap was set to $400 \mu \mathrm{m}$. Nucleation density was calculated indirectly from the images by 
converting the number of spherulites on a certain area $\mathrm{N}_{\mathrm{A}}$, to the number of spherulites per unit volume $\mathrm{N}_{\mathrm{V}}$, by means of Eq. (1). This formula can only be used when all spherulites are of identical size [1].

$$
N_{V}=(N A)^{\frac{3}{2}}
$$

Rheological measurements were performed using an advanced rheometrics expansion system (ARES rheometer, TA instruments), in nitrogen atmosphere. The diameter of the parallel plate geometry was $25 \mathrm{~mm}$ and the measurement gap was equal to $1.4 \mathrm{~mm}$. Sample discs used for the measurement were prepared by molding dried PLA pellets or strings at $200^{\circ} \mathrm{C}$ and $50 \mathrm{bar}$, for $4 \mathrm{~min}$, in a Collin laboratory press. Crystallization behavior was investigated by using an oscillatory time sweep test. The zero shear viscosity of the samples was determined with frequency sweeps in the linear viscoelastic range $\left(200^{\circ} \mathrm{C}, 5 \%\right.$ deformation).

GPC-IR (Polymer Char) was used to determine molecular weight and molecular weight distribution with respect to polystyrene standards. To this end, samples were dissolved in dichlorobenzene.

A differential scanning calorimeter, Q2000 (TA instruments), was used to determine thermal transitions of samples which were loaded into aluminum Tzero DSC pans and heated according to the isothermal crystallization protocol explained in the protocols and analyses section. Sample masses ranged between $5 \mathrm{mg}$ and $10 \mathrm{mg}$.

\subsection{Protocols and analyses}

Isothermal crystallization experiments were performed with an ARES rheometer, a DSC and the Linkam shearing

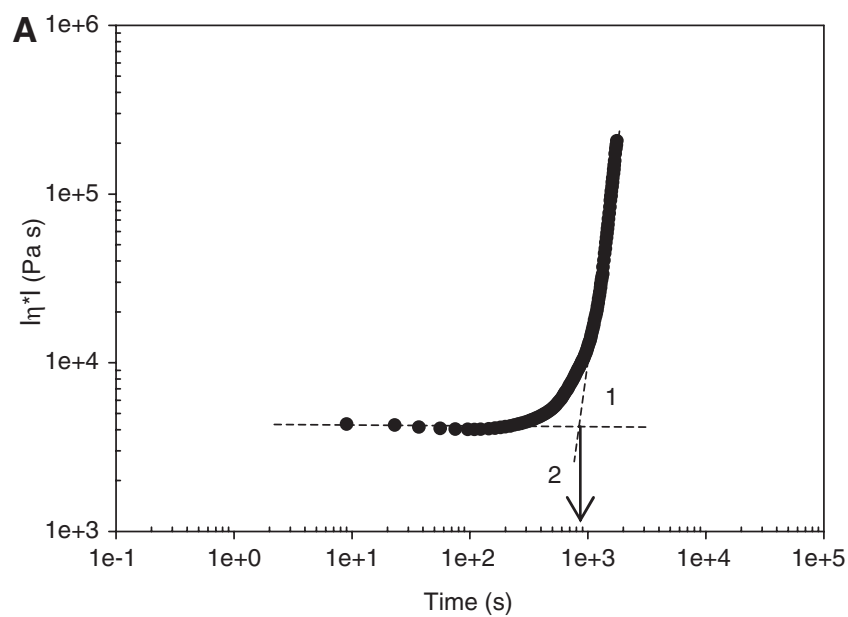

cell in conjunction with optical microscopy. All samples used for these experiments were subjected to the following isothermal crystallization protocol: heating from $40^{\circ} \mathrm{C}$ to the annealing temperature of $200^{\circ} \mathrm{C}$ at $10^{\circ} \mathrm{C} \mathrm{min} \mathrm{m}^{-1}$ followed by an isotherm of $10 \mathrm{~min}$ to erase the thermo-mechanical history. Subsequently, the sample was cooled at $10^{\circ} \mathrm{C} \mathrm{min}^{-1}$ to the $\mathrm{T}_{\mathrm{c}}$, in this study equal to $130^{\circ} \mathrm{C}$, where crystallization was monitored up to a stage that was allowed by the instrument (ARES rheometer) or up to completion (DSC, optical microscopy). In the case of crystallization experiments performed with the ARES rheometer, the samples were cooled at $5^{\circ} \mathrm{C} \mathrm{min}^{-1}$ due to instrumental limitations.

Rheological results were represented by the modulus of complex viscosity and for DSC by the exothermal heat flow, both as functions of time. From these curves, characteristic crystallization times can be defined. In the case of rheology, the characteristic time corresponds to the time at which lines 1 and 2 intercept, see Figure 1A. In DSC, the characteristic crystallization time was defined as the time at which the heat flow peak reaches its maximum (Figure 1B).

The Lotz analysis [34] has been widely used in the literature to address the nucleation efficiency of additives. In this analysis, the nucleation efficiency of a given nucleating agent in a polymeric material is compared to an "ideally" nucleated polymer. The latter can be achieved by using self-nucleation, since in terms of dispersion, chemical interaction and crystallographic matching, crystal nuclei of the polymer itself are the ideal agent.

In literature, as well as in industry, Eq. (2) is currently used to calculate the nucleation efficiency $[14,15,22$, 35-37] .

$$
N E=100 \frac{\mathrm{T}_{c, 1}-\mathrm{T}_{c}}{\mathrm{~T}_{\mathrm{c}, \max }-\mathrm{T}_{c}}=100 \frac{\Delta \mathrm{T}_{N E}}{\Delta \mathrm{T}}
$$

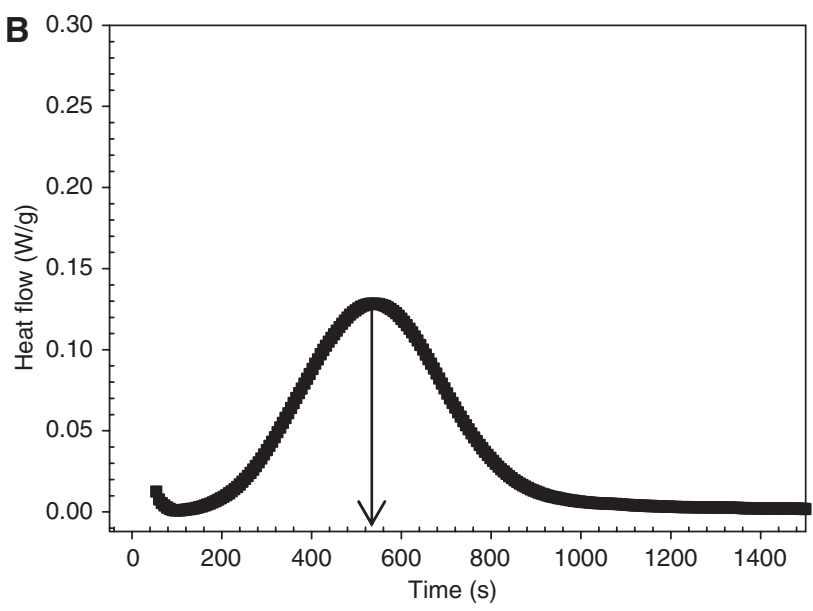

Figure 1 Determination of characteristic crystallization time: (A) rheology, (B) calorimetry. 
Here, $T_{c}$ is the crystallization temperature of the pure polymer (lower limit), $\mathrm{T}_{c, 1}$ the crystallization temperature of the polymer nucleated with nucleating agent 1 , and $\mathrm{T}_{c, \max }$ the crystallization temperature of the self-nucleated polymer (upper limit).

In the Lotz analysis, the crystallization temperature of the pure and nucleated polymers are usually determined from non-isothermal crystallization DSC experiments. The $\mathrm{T}_{\mathrm{c}}$ of the self-nucleated polymer is obtained by following the temperature protocol below and repeating it at different nucleating temperatures (Figure 2A). During this protocol, the polymer is partially melted and residual crystals are used as "ideal" nucleating agents. The concentration of residual crystals depends on the self-nucleating temperature (i.e., the upper temperature used during partial melting) and partial melting period. Figure 2B shows that, after partial melting above a certain temperature, almost no residual crystals are left to facilitate crystallization. If the self-nucleating temperature decreases, the concentration increases, affecting the $\mathrm{T}_{c}$. At a certain self-nucleation temperature, the concentration reaches a saturation point and the corresponding $\mathrm{T}_{\mathrm{c}}$ reaches its maximum which is equal to $\mathrm{T}_{\mathrm{c} \text { max }}$.

For PLA used in this work, the self-nucleating or selfseeding temperature was varied from $165^{\circ} \mathrm{C}$ to $174^{\circ} \mathrm{C}$ and each partial melting period lasted $10 \mathrm{~min}$. These temperatures are situated between the dotted lines depicted in Figure $2 \mathrm{~A}$. The highest $\mathrm{T}_{c}$, equal to $157^{\circ} \mathrm{C}$, was measured at a self-nucleation temperature of $168^{\circ} \mathrm{C}$ while cooling at $10^{\circ} \mathrm{C} \min ^{-1}$.

Despite the fact that many industrial polymer processing techniques use a non-isothermal protocol, isothermal conditions are favored in laboratory studies mainly due to practical reasons. This is especially the case when rheology is involved, as achieving non-isothermal conditions in a rheometer is problematic. Using a constant temperature limits thermal gradients within samples, making the comparison of results obtained by different techniques straightforward, since no dependencies, for instance on cooling rates, are involved. Classical non-isothermal Lotz analysis cannot be used to compare isothermal crystallization DSC experiments. Accordingly, in this work, an alternative analysis method is proposed in which crystallization times rather than temperatures are used. Calculation of isothermal nucleating efficiency can be performed with Eq. (3), with $t_{c}$ the crystallization time of the pure polymer (upper limit), $t_{c, 1}$ the crystallization time of the polymer nucleated with nucleating agent 1 and $t_{c, \text { min }}$ the crystallization time of the self-nucleated polymer (lower limit).

$$
N E=100 \frac{\mathrm{t}_{\mathrm{c}}-\mathrm{t}_{\mathrm{c}, 1}}{\mathrm{t}_{\mathrm{c}}-\mathrm{t}_{\mathrm{c}, \min }}=100 \frac{\Delta \mathrm{t}_{N A}}{\Delta \mathrm{t}}
$$

The crystallization time of the pure and nucleated polymer are obtained from isothermal crystallization DSC experiments using the isothermal crystallization protocol described above. The crystallization time of the self-nucleated polymer is determined by following the temperature protocol shown in Figure 3 and repeating it at different self-nucleating temperatures. The nucleation temperatures $\left(165^{\circ} \mathrm{C}-174^{\circ} \mathrm{C}\right)$ and period $(10 \mathrm{~min})$ are the same as in the non-isothermal case, but rather than cooling samples to a temperature below the glass transition temperature, they remain at the chosen $\mathrm{T}_{c}$. Consequently, the material crystallizes isothermally, instead of non-isothermally. The $\mathrm{T}_{\mathrm{c}}$ was selected to be $130^{\circ} \mathrm{C}$.
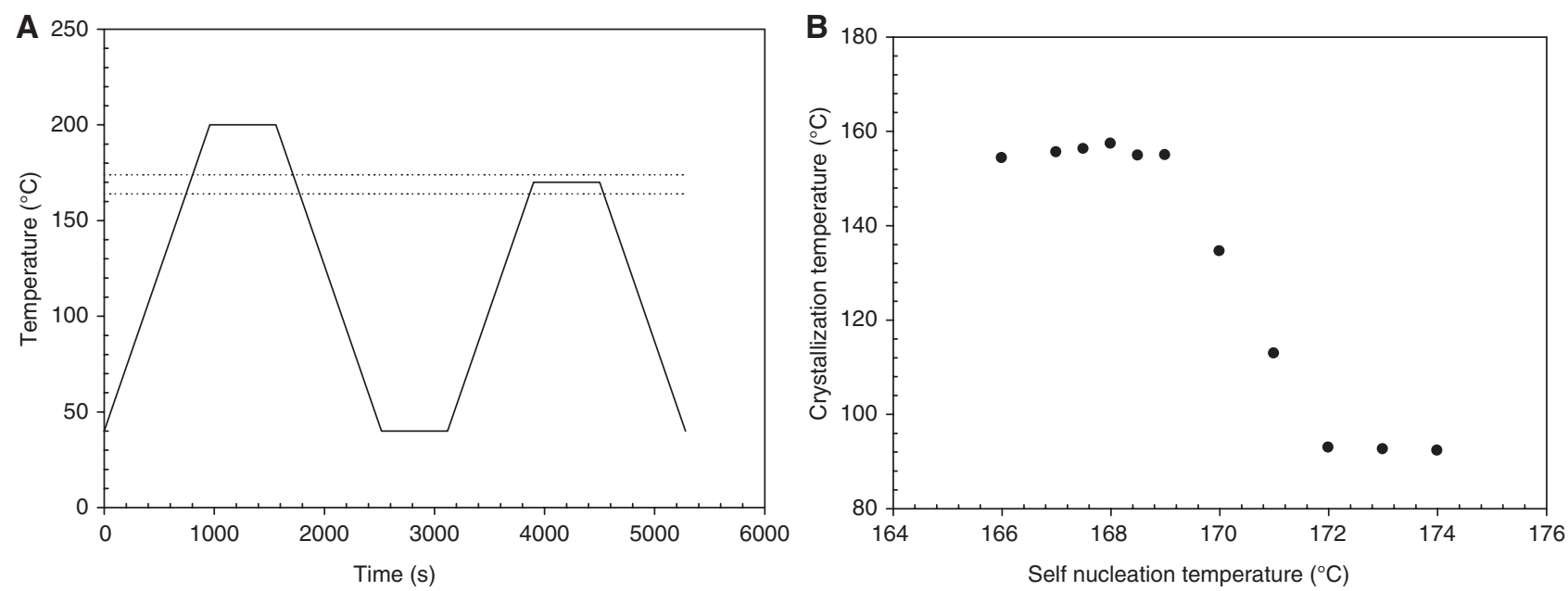

Figure 2 Protocol of the non-isothermal Lotz analysis (A) and crystallization temperature as a function of self nucleation temperature (B). 


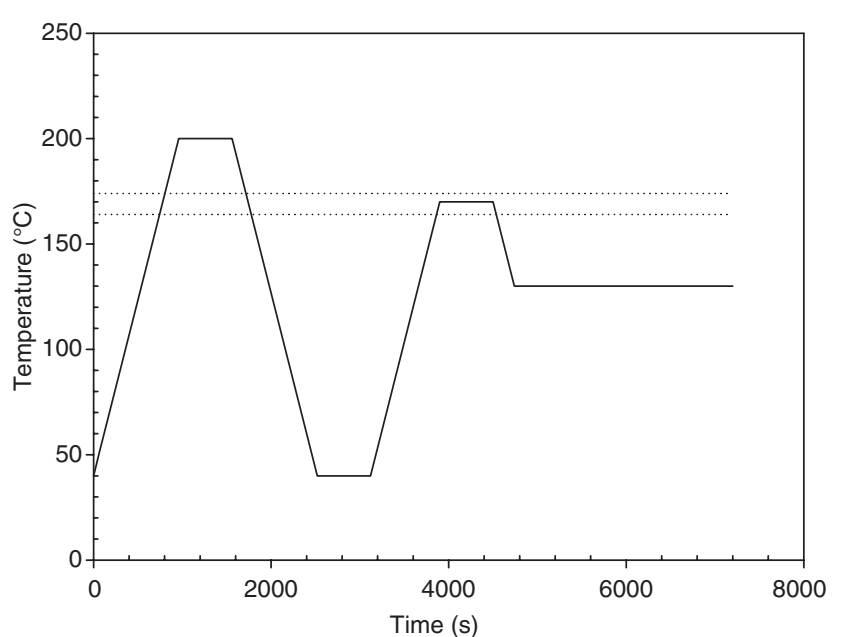

Figure 3 Protocol of the isothermal Lotz analysis. The nucleation temperature was varied from $165^{\circ} \mathrm{C}$ to $174^{\circ} \mathrm{C}$, depicted between dotted lines.

As in the non-isothermal case, the crystallization behavior of self-nucleated samples depends on the selfnucleation temperature since as it decreases, the residual crystal concentration increases and crystallization time is reduced. The non-isothermal analysis showed that the concentration of residual crystals is saturated when the nucleation temperature is equal to $168^{\circ} \mathrm{C}$. Under normal isothermal conditions, the crystallization time corresponding to this saturation point is difficult to measure, since the polymer crystallization is faster than the cooling rate and thus heat flow reaches its maximum $-155 \mathrm{~s}$ before reaching the isotherm, implicating a negative value for $\mathrm{t}_{c, \text { min }}$, when cooling at $10^{\circ} \mathrm{C} \mathrm{min}^{-1}$. Increasing the cooling rate was no option, as the heat flow signal during the crystallization process was easily affected by overshoots. The proposed method to obtain a value for the self-nucleated crystallization is to shift the time scale used for the isothermal nucleating efficiency calculation: the time 0 was moved from the starting point of the isotherm to the starting point of the cooling zone from $168^{\circ} \mathrm{C}$, being a difference of $228 \mathrm{~s}$. In this case, $t_{c, \min }$ was equal to $73 \mathrm{~s}$.

\section{Results and discussion}

As explained in the introduction, the isothermal crystallization behavior of PLA is too slow for industrial use. Moreover, additives which do not compromise PLA bio-advantages are preferred to modify its properties. Therefore, the present work improves the isothermal crystallization behavior of PLA by introducing amino acids and poly(amino acids) such as glycine, L-alanine, polyglycine and poly-DL-alanine as heterogeneous nucleating agents. The discussion is based on the results obtained for polyglycine. Data related to the other nucleating agents can be found in the supporting information section (Table A1 and A2).

\subsection{Effect of diverse nucleating agents on the isothermal crystallization of PLA}

The isothermal crystallization behavior of PLA, aided by different nucleating agents, was studied at $130^{\circ} \mathrm{C}$. According to literature $[8,9]$, the formed crystal structure at this $\mathrm{T}_{\mathrm{c}}$ is the $\alpha$-form. To compare the effect of different agents, they were added at the same concentration: $1.5 \mathrm{wt} \%$.

Rheology was used to monitor the evolution of crystallization degree during isothermal crystallization (Figure 4). Samples were cooled from the melt at $5^{\circ} \mathrm{C} \mathrm{min}^{-1}$ after which time sweep tests were performed at $130^{\circ} \mathrm{C}$. During the small amplitude oscillatory experiments, the material was deformed with a sinusoidal trend, with a frequency equal to $1 \mathrm{rad} \mathrm{s}^{-1}$, and deformation of $5 \%$.

During the isothermal crystallization step, the modulus of complex viscosity normalized with its value at time 0 was observed as a function of time. This type of curve usually has a sigmoidal form, meaning that the start of the curve corresponds to a low constant value, as long as no crystals are present, after which the viscosity suddenly increases due to the growing spherulites. Finally, when the sample is totally solidified, the viscosity reaches a constant value higher than the initial one. Since equipment limits were reached, by cause of a combination of high viscosities and resonance frequencies, the

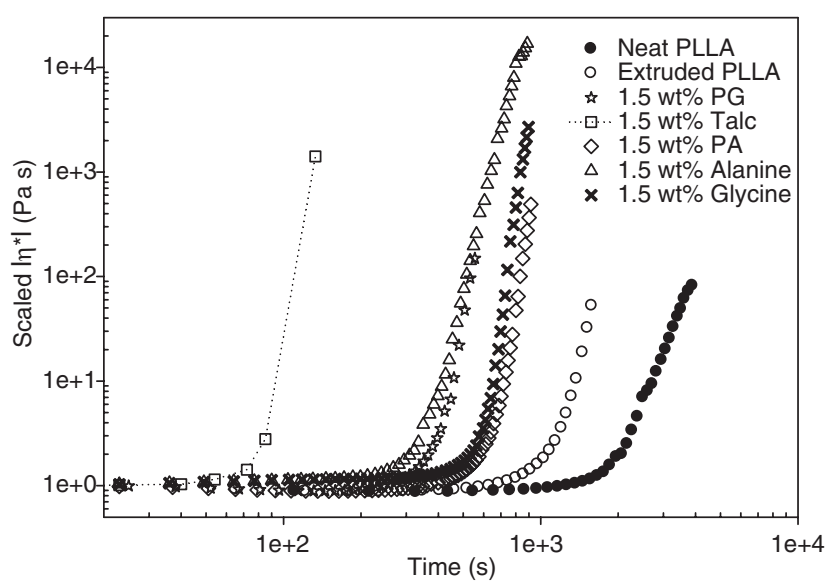

Figure 4 Evolution of the modulus of complex viscosity in time, obtained from rheological isothermal crystallization experiments at $130^{\circ} \mathrm{C}$. The modulus of the complex viscosity was scaled with its value at time 0 . 


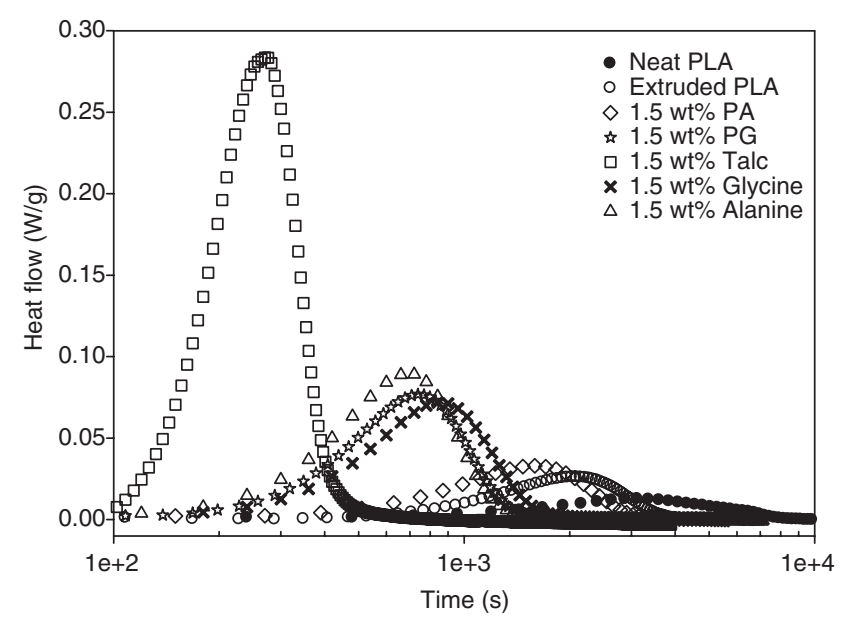

Figure 5 Evolution of heat flux in time, obtained from DSC isothermal crystallization experiments at $130^{\circ} \mathrm{C}$. The exothermic axis is plotted upwards.

experiments were stopped before this last zone could be observed.

The calorimetric graph shows the exothermic heat flow released during the crystallization process as a function of time (Figure 5).

The characteristic crystallization times determined from the curves in Figures 4 and 5, together with the isothermal nucleating efficiency based on DSC experiments and calculated using Eq. (3) can be found in Table 3.

The results from both experimental techniques present the same trend: the values corresponding to samples containing nucleating agents are shifted to shorter times with respect to the extruded neat material, as the crystallization process is accelerated. The effect of the extrusion step is reflected in the shift of the extruded PLA curves with respect to non-extruded PLA, termed neat PLA. The results indicate that talc is the best nucleating agent, but

Table 3 Comparison of the characteristic crystallization times obtained from rheological and calorimetrical isothermal crystallization experiments. The DSC isothermal nucleating efficiency was calculated respect to extruded neat PLA.

\begin{tabular}{lrrr}
\hline $\begin{array}{l}\text { Nucleating } \\
\text { agent }\end{array}$ & $\begin{array}{r}\text { Rheology } \\
\text { (s) }\end{array}$ & $\begin{array}{r}\text { Calorimetry } \\
(\mathbf{s})\end{array}$ & $\begin{array}{r}\text { Nucleating } \\
\text { efficiency (\%) }\end{array}$ \\
\hline None: neat PLA & 1857 & 3136 & - \\
None: extruded PLA & 1123 & 2115 & - \\
L-alanine & 340 & 694 & 62.6 \\
Poly-DL-alanine & 576 & 1605 & 22.5 \\
Glycine & 598 & 886 & 54.1 \\
Polyglycine & 380 & 741 & 60.5 \\
Talc & 93 & 273 & 81.1 \\
\hline
\end{tabular}

polyglycine and L-alanine also exhibit a significant effect. Poly-DL-alanine and glycine show minor effects, but still induce some acceleration of crystallization.

The isothermal crystallization behavior was also monitored by polarized light microscopy. With this method, the microstructure of neat PLA and the nucleated samples could be directly compared (Figure 6). It has already been highlighted that the nucleation density of neat PLA is very low, which is confirmed by the microscopy picture in Figure 6A, showing a lonely spherulite. The samples of extruded PLA present a higher nucleation density which still increases significantly when talc was added. In the same fashion, the proposed alternative nucleating agents exhibit a significant effect.

Moreover, the microscopic pictures were utilized to obtain quantitative information regarding the nucleation density, calculated from Eq. (1). The values in Table 4 confirm what was already deduced by visual analysis of the microscopic photographs: the nucleation density increases when adding nucleating agents, nevertheless in different magnitudes. This shows that there is a correlation between the crystallization acceleration, measured on the nucleating efficiency scaled, and the increase in nucleation density.

\subsection{Effect of nucleating agents concentration on the isothermal crystallization of PLA}

The effect of varying the concentration of nucleating agents on PLA crystallization was studied with L-alanine, poly-DL-alanine, polyglycine and talc. Polyglycine and alanine showed the highest nucleating efficiency, after talc. Polyglycine was chosen as an example for the study of crystallization behavior as a function of nucleating agent concentration. The use of alanine also has a negative effect on the mechanical properties of the samples because of the huge difference in molecular weight between PLA and alanine, being a decrease of viscosity and increase of brittleness.

Figure 7 shows the evolution of the modulus of complex viscosity during the isothermal crystallization of PLA nucleated with different concentrations of polyglycine. In Figure 8, the isothermal crystallization DSC curves are compared. The values corresponding to samples containing higher concentrations of nucleating agents are shifted to shorter times with respect to the extruded and neat PLA, as the crystallization process is accelerated.

From this set of experiments, and the homologue ones corresponding to the other nucleating agents, the 


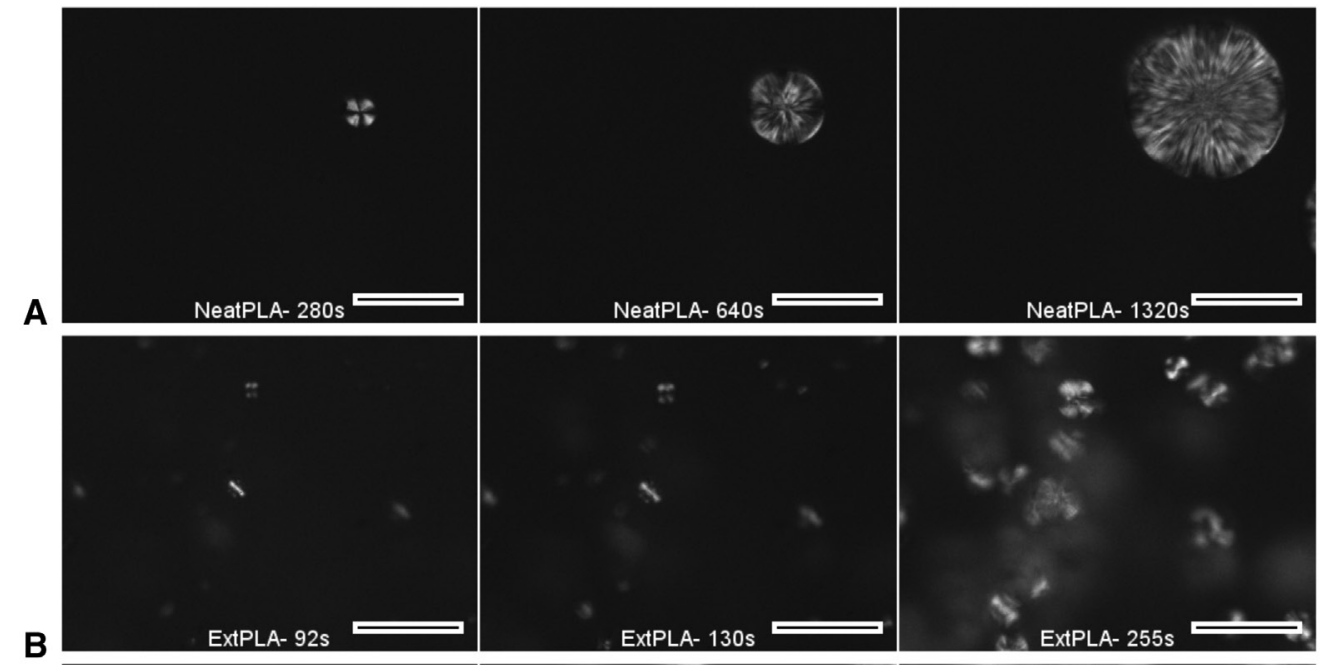

B
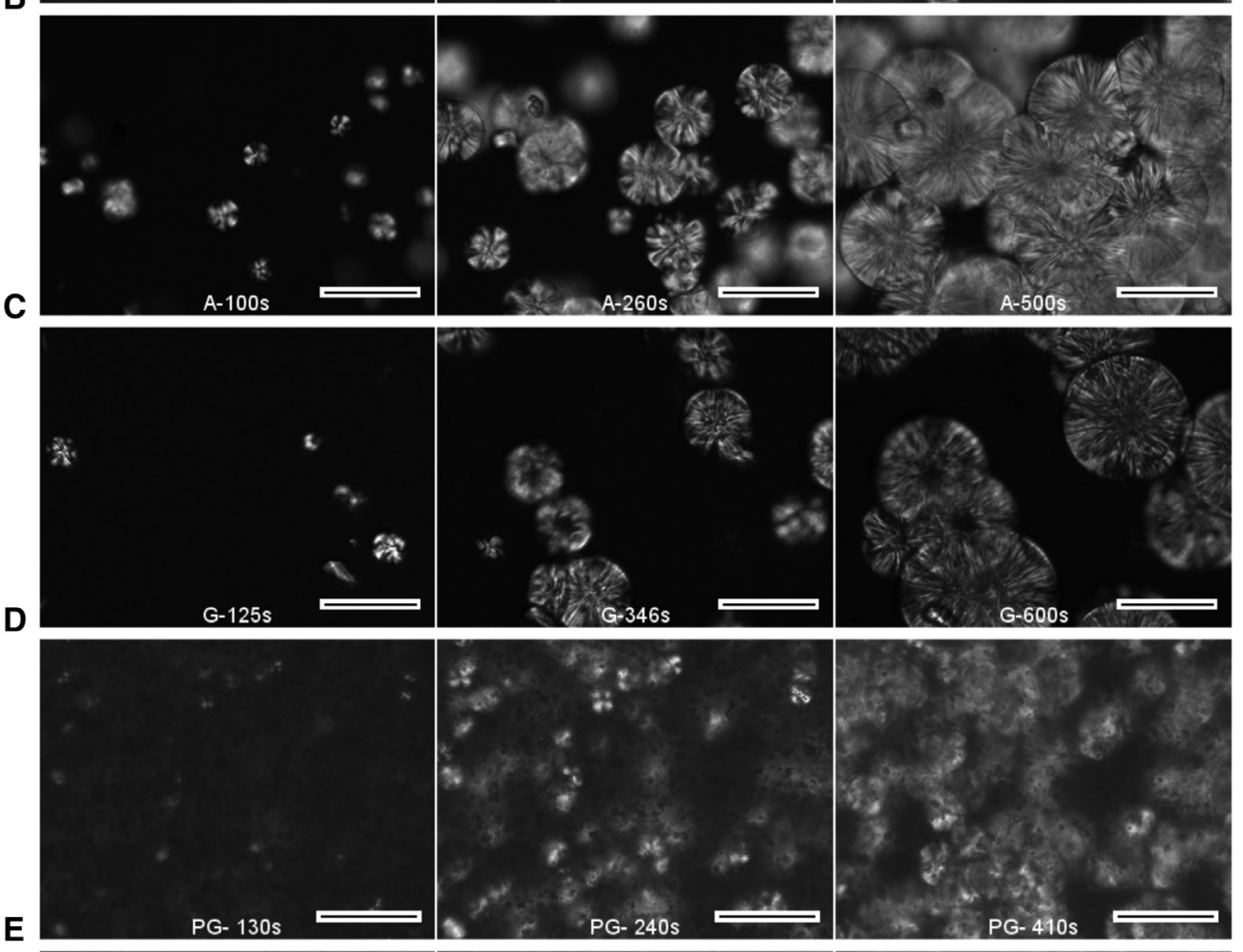

E
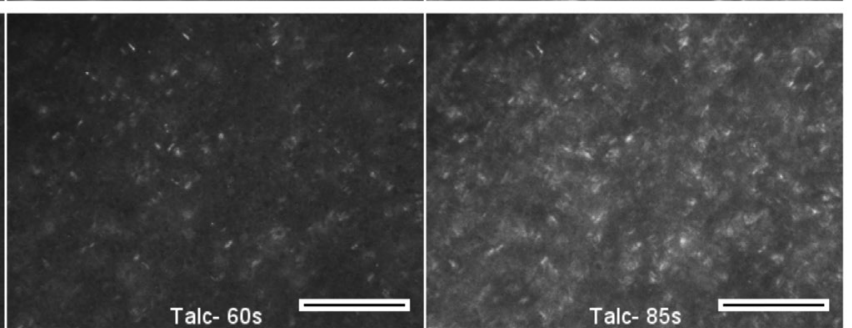

F Talc- $38 \mathrm{~s}$

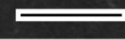

Talc- 60 s

Figure 6 Morphology evolution during isothermal crystallization experiments at $130^{\circ} \mathrm{C}$. The scale line is $100 \mu \mathrm{m}$. (A) neat PLA, (B) extruded PLA, (C) PLA+1.5 wt\% L-alanine, (D) PLA+1.5 wt\% glycine, (E) PLA+1.5 wt\% polyglycine, (F) PLA+1.5 wt \% talc.

characteristic crystallization times were determined and compiled in Figures 9 and 10, where the characteristic crystallization time as a function of nucleating agent concentration is depicted. The results obtained from rheology and calorimetry were in agreement, except for polyDL-alanine. The highest crystallization times correspond 
Table 4 Nucleating density from isothermal crystallization experiments at $130^{\circ} \mathrm{C}$.

\begin{tabular}{|c|c|}
\hline Nucleating agent & $\begin{array}{r}\text { Nucleating density } \\
\text { (nuclei } \mathrm{m}^{-3} \text { ) }\end{array}$ \\
\hline None: neat PLA & $1.8110^{7}$ \\
\hline None: extruded PLA & $1.5010^{8}$ \\
\hline PLA+1.5 wt $\%$ L-alanine & $4.1010^{8}$ \\
\hline PLA+1.5 wt\% glycine & $2.2910^{8}$ \\
\hline PLA+1.5 wt $\%$ polyglycine & $3.1610^{9}$ \\
\hline PLA+1.5 wt $\%$ talc & $4.9910^{10}$ \\
\hline
\end{tabular}

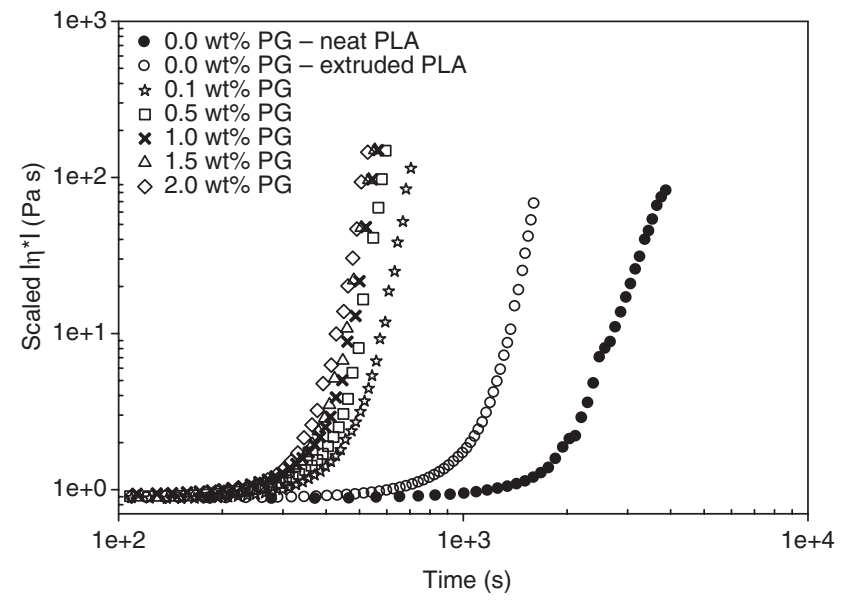

Figure 7 Evolution of the modulus of complex viscosity in time, obtained from isothermal crystallization experiments of poly(lactic acid) (PLA) samples with different concentrations of polyglycine (0.0 wt $\%, 0.1 \mathrm{wt} \%, 0.5 \mathrm{wt} \%, 1.0 \mathrm{wt} \%, 1.5 \mathrm{wt} \%, 2.0 \mathrm{wt} \%)$. The crystallization temperature was $130^{\circ} \mathrm{C}$. The modulus of the complex viscosity was scaled with its value at time 0 .

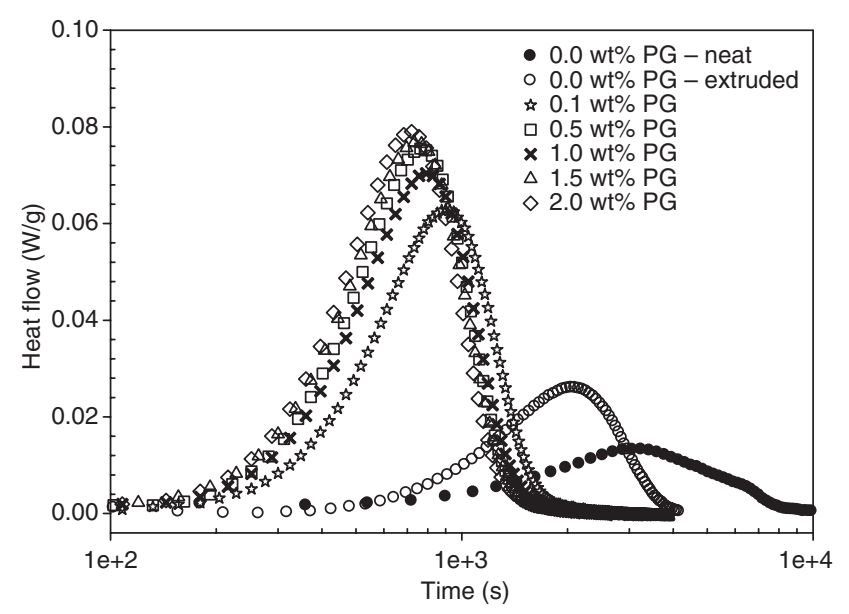

Figure 8 Evolution of heat flow in time, obtained from isothermal crystallization experiments of poly(lactic acid) (PLA) samples with different concentrations of polyglycine $(0.0 \mathrm{wt} \%, 0.1 \mathrm{wt} \%, 0.5 \mathrm{wt} \%$, $1.0 \mathrm{wt} \%, 1.5 \mathrm{wt} \%, 2.0 \mathrm{wt} \%)$. The crystallization temperature was $130^{\circ} \mathrm{C}$. The exothermic axe is plotted upwards.

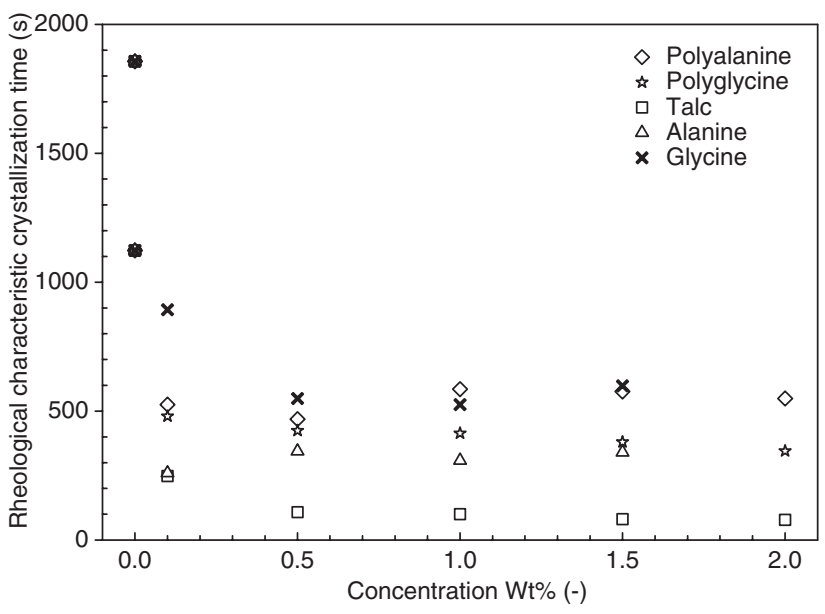

Figure 9 Comparison of the rheological characteristic crystallization time of samples with different nucleating agents as a function of concentration (0.0 wt \%, $0.1 \mathrm{wt} \%, 0.5 \mathrm{wt} \%, 1.0 \mathrm{wt} \%, 1.5 \mathrm{wt} \%, 2.0$ $w t \%)$.

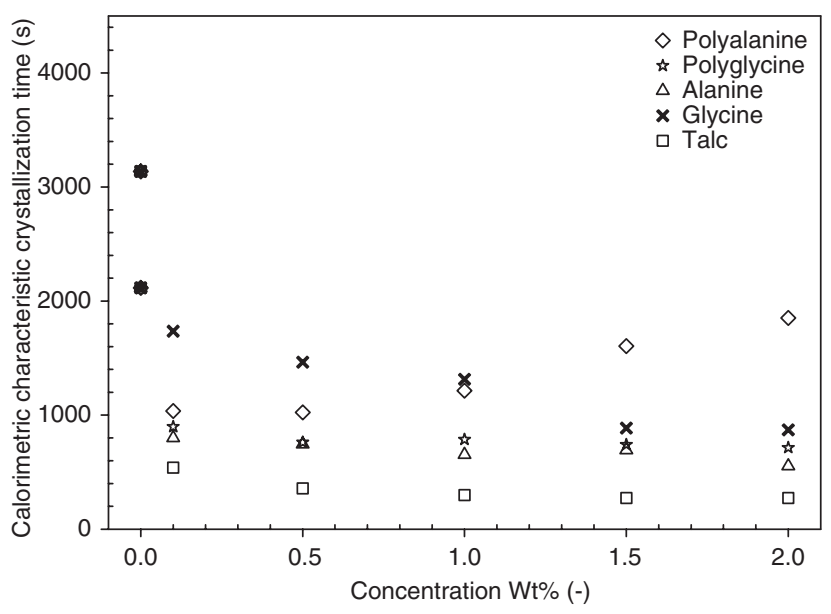

Figure 10 Comparison of the calorimetric characteristic crystallization time of samples with different nucleating agents as a function of concentration (0.0 wt \%, $0.1 \mathrm{wt} \%, 0.5 \mathrm{wt} \%, 1.0 \mathrm{wt} \%, 1.5 \mathrm{wt} \%, 2.0$ $w t \%)$.

to the neat PLA and the extruded neat PLA samples, represented at $0.0 \mathrm{wt} \%$ concentration. The addition of the chosen biomolecules decreased the crystallization time of PLA until a saturation point, followed by a plateau. In the case of polyglycine, L-alanine and poly-DL-alanine, this critical value was lower than the minimum concentration used in this work, while for talc and glycine a decreasing trend can still be observed at the measured concentrations.

The effect of poly-DL-alanine concentration was also measured with both techniques. According to rheology, the addition of poly-DL-alanine to PLA has the same effect as the other nucleating agents. By contrast, 
A
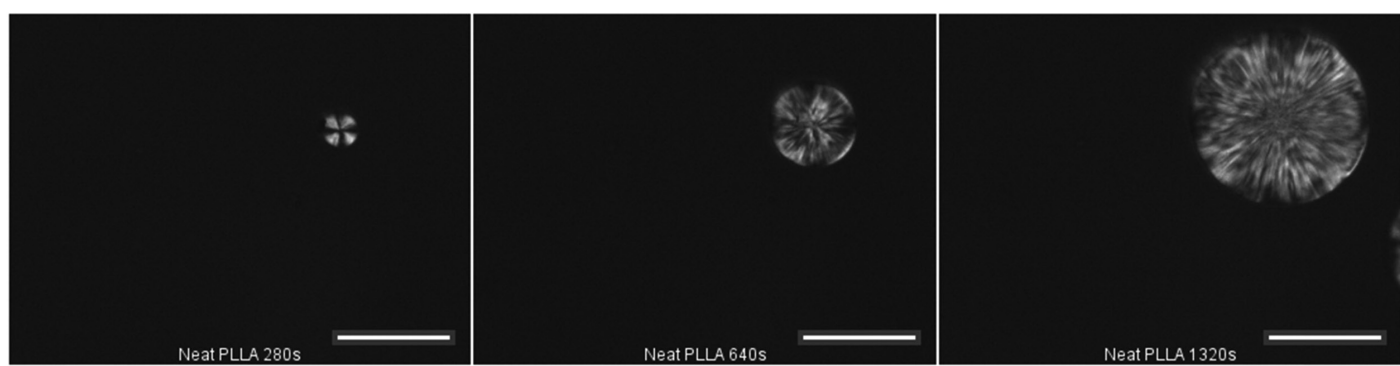

B
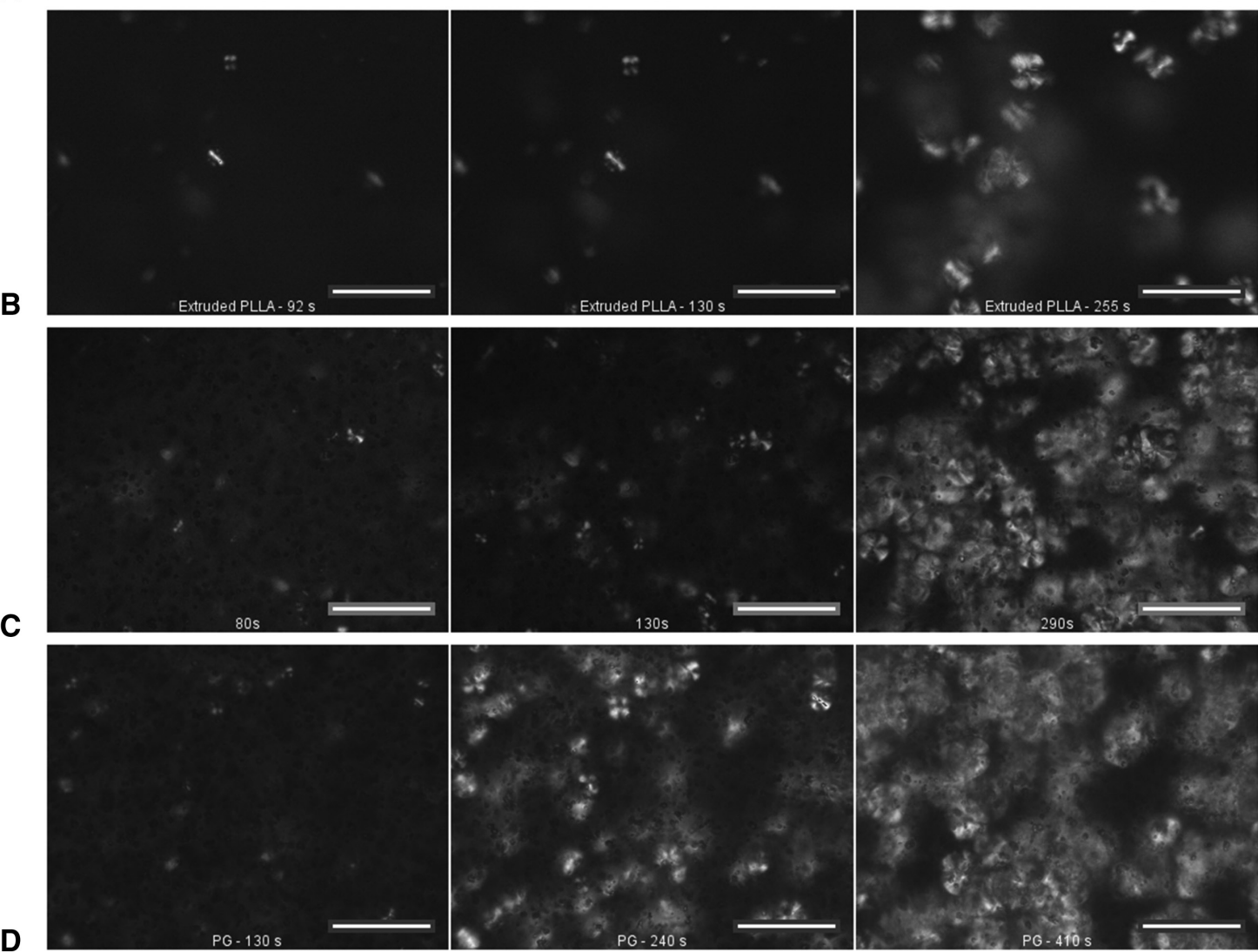

Figure 11 Morphology evolution during isothermal crystallization experiments at $130^{\circ} \mathrm{C}$. The scale line is $100 \mu \mathrm{m}$. PLA+polyglycine [0.0 wt \% neat (A) and extruded (B), $0.5 \mathrm{wt} \%(\mathrm{C}), 1.5 \mathrm{wt} \%(\mathrm{D})]$.

the calorimetric results show that if the concentration keeps increasing after the crystallization time reached a minimum, it starts increasing again. A possible explanation for the discrepancy is the difference in masses used for each technique, since those used for rheology were significantly larger than the ones for calorimetry, herewith gaining information of average properties and thus making mixing during sample preparation a key factor. It was noted that the poly-DL-alanine particles were easily electrostatically charged, making handling more complicated and homogeneity more difficult to ensure.

The use of amino acids as nucleating agents has some disadvantages with respect to using poly(amino acids) or polypeptides. The addition of low molecular weight molecules caused a considerable decrease of the shear viscosity and melting temperature (Figure A1). Moreover, this was coupled with an increased brittleness. It can be presumed that the use of other low molecular weight biomolecules could lead to the same kind of problems, while the addition of the poly(amino acids) did not face such kinds of drawbacks because of the higher molecular weight.

In addition to calorimetric and rheological measurements, a microscopic study was performed. From these results, the trend with polyglycine samples is clear and agrees with the previous conclusions. The acceleration of PLA crystallization as polyglycine concentration increases is related to a higher amount of nucleation sites (Figure 11). 


\section{Conclusions}

The isothermal crystallization behavior of PLA nucleated with different amino and poly(amino acids) was studied at various concentrations by using diverse measurement techniques, such as calorimetry, rheology and polarized light microscopy. Hereby, the crystallization process was assessed on various levels with independent means. The nucleating effect of the proposed and studied amino acids and poly(amino acids) was confirmed. In the case of polyglycine, low concentrations were sufficient to obtain a significant improvement without compromising PLA bioadvantages and mechanical properties.

\section{Appendix 1 Supporting information.}

Table A1 Comparison of the characteristic crystallization times obtained from rheological isothermal crystallization experiments.

\begin{tabular}{|c|c|c|c|c|c|c|}
\hline \multirow[t]{2}{*}{ Nucleating agent } & \multirow[b]{2}{*}{$0.0 \mathrm{wt} \%$} & \multirow[b]{2}{*}{$0.1 \mathrm{wt} \%$} & \multirow[b]{2}{*}{$0.5 \mathrm{wt} \%$} & \multicolumn{3}{|c|}{ Rheological characteristic crystallization time } \\
\hline & & & & 1.0 wt $\%$ & $1.5 \mathrm{wt} \%$ & $2.0 \mathrm{wt} \%$ \\
\hline Neat poly(lactic acid) & 1857 & - & - & - & - & - \\
\hline Extruded poly(lactic acid) & 1123 & - & - & - & - & - \\
\hline L-alanine & - & 260 & 345 & 309 & 340 & - \\
\hline Poly-DL-alanine & - & 525 & 469 & 585 & 576 & 549 \\
\hline Glycine & - & 893 & 549 & 525 & 598 & - \\
\hline Polyglycine & - & 480 & 424 & 414 & 380 & 345 \\
\hline Talc & - & 248 & 108 & 100 & 93 & 78 \\
\hline
\end{tabular}

Table A2 Comparison of the characteristic crystallization times obtained from calorimetrical isothermal crystallization experiments.

\begin{tabular}{|c|c|c|c|c|c|c|}
\hline \multirow[t]{2}{*}{ Nucleating agent } & \multicolumn{6}{|c|}{ Calorimetrical characteristic crystallization time } \\
\hline & 0.0 wt $\%$ & 0.1 wt $\%$ & $0.5 \mathrm{wt} \%$ & 1.0 wt $\%$ & $1.5 w t \%$ & $2.0 \mathrm{wt} \%$ \\
\hline Neat poly(lactic acid) & 3136 & - & - & - & - & - \\
\hline Extruded poly(lactic acid) & 2115 & - & - & - & - & - \\
\hline L-alanine & - & 800 & 745 & 653 & 694 & 553 \\
\hline Poly-DL-alanine & - & 1036 & 1023 & 1213 & 1605 & 1851 \\
\hline Glycine & - & 1736 & 1464 & 1314 & 886 & 871 \\
\hline Polyglycine & - & 899 & 761 & 785 & 741 & 715 \\
\hline Talc & - & 539 & 357 & 299 & 273 & 272 \\
\hline
\end{tabular}

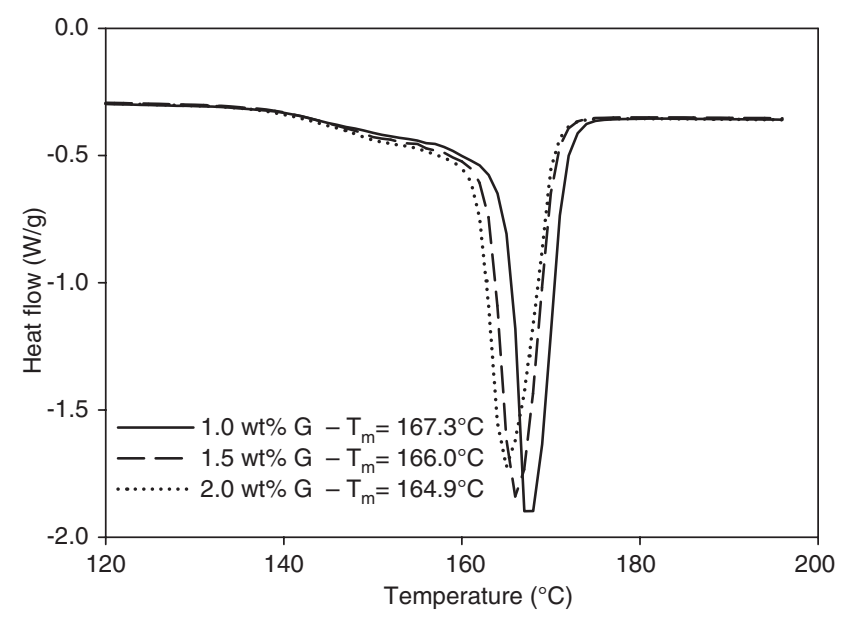

Figure A1 Effect of the concentration of glycine on the melting temperature of PLA.

\section{References}

[1] Janeschitz-Kriegl H. Crystallization Modalities in Polymer Melt Processing: Fundamental Aspects of Structure Formation, Springer-Verlag Wien: Germany, 2010.

[2] Reiter G, Strobl GR. Progress in Understanding of Polymer Crystallization, Springer: Germany, 2007.

[3] Lunt J. Polym. Degrad. Stab. 1998, 59, 145-152.

[4] Jamshidian M, Tehrany EA, Imran M, Jacquot M, Desobry S. Compr. Rev. Food Sci. Food Saf. 2010, 9, 552-571.

[5] Rasal RM, Janorkar AV, Hirt DE, Prog. Polym. Sci. 2010, 35, 338-356.

[6] Lim LT, Auras R, Rubino M. Prog. Polym. Sci. 2008, 33, 820-852.

[7] Iannace S, Nicolais L. J. Appl. Polym. Sci. 1997, 64, 911-919.

[8] Di Lorenzo ML. Eur. Polym. 2005, 41, 569-575.

[9] Yasuniwa M, Tsubakihara S, lura K, Ono Y, Dan Y, Takahashi K. Polymer 2006, 47, 7554-7563. 
[10] Miyata T, Masuko T. Polymer 1998, 39, 5515-5521.

[11] He Y, Fan Z, Hu Y, Wu T, Wei J, Li S. Eur. Polym. J. 2007, 43, 4431-4439.

[12] Huang J, Lisowski MS, Runt J, Hall ES, Kean RT, Buehler N, Lin JS. Macromolecules 1998, 31, 2593-2599.

[13] Tsuji H, Tezuka Y, Saha S K, Suzuki M, Itsuno S. Polymer 2005, 46, 4917-4927.

[14] Anderson KS, Hillmyer MA. Polymer 2006, 47, 2030-2035.

[15] Schmidt SC, Hillmyer MA. J. Polym. Sci. Part B Polym. Phys. 2001, 39, 300-313.

[16] Yamane H, Sasai K. Polymer 2003, 44, 2569-2575.

[17] Tsuji H, Takai H, Fukuda N, Takikawa H. Macromol. Mater. Eng. 2006, 291, 325-335.

[18] Tsuji H, Takai H, Saha SK. Polymer 2006, 47, 3826-3837.

[19] Rahman N, Kawai T, Matsuba G, Nishida K, Kanaya T, Watanabe H, Okamoto H, Kato M, Usuki A, Matsuda M, Nakajima K, Honma N. Macromolecules 2009, 42, 4739-4745.

[20] Narita J, Katagiri M, Tsuji H. Macromol. Mater. Eng. 2011, 296, 887-893.

[21] Xiao HW, Li P, Ren X, Jiang T, Yeh J. J. Appl. Polym. Sci. 2010, 118, 3558-3569.

[22] Kolstad J. J. Appl. Polym. Sci. 1996, 62, 1079-1091.

[23] Bai H, Zhang W, Deng H, Zhang Q, Fu Q. Macromolecules 2011, 44, 1233-1237.
[24] Liao R, Yang B, Yu W, Zhou C. J. Appl. Polym. Sci. 2007, 104, 310-317.

[25] Kawamoto N, Sakai A, Horikoshi T, Urushihara T, Tobita E. J. Appl. Polym. Sci. 2007, 103, 198-203.

[26] Harris AM, Lee EC. J. Appl. Polym. Sci. 2008, 107, 2246-2255.

[27] Tsuji H, Tashiro K, Bouapao L, Narita J. Macromol. Mater. Eng. 2008, 293, 947-951.

[28] Tsuji H, Sawada M, Bouapao L. ACS Appl. Mater. Interfaces 2009, 1, 1719-1730.

[29] Pan P, Yang J, Shan G, Bao Y, Weng Z, Inoue Y. Macromol. Mater. Eng. 2012, 297, 670-679.

[30] Tachibana Y, Maeda T, Ito O, Maeda Y, Kunioka M. Polym. Degrad. Stab. 2010, 95, 1321-1329.

[31] Pei A, Zhou Q, Berglund LA. Compos. Sci. Technol. 2010, 70, 815-821.

[32] Boeckx B, Doctoral Thesis, KU Leuven (Belgium), March 2011.

[33] Vanhalle M, Master Thesis, KU Leuven (Belgium), June 2010.

[34] Fillon B, Thierry A, Lotz B, Wittman JC. J. Therm. Anal. 1994, 42, 721-731.

[35] D'Haese M, Van Puyvelde P, Langouche F. Macromolecules 2010, 43, 2933-2941.

[36] D’Haese M, Goderis B, Van Puyvelde P. Macromol. Mater. Eng. 2011, 296, 603-616.

[37] D'Haese M, Langouche F, Van Puyvelde P. Macromolecules 2013, $463425-3434$. 